\title{
Application of the Wigner-Ville Distribution to Temperature Gradient Microstructure: A New Technique to Study Small-Scale Variations
}

\author{
JORG IMBERGER* \\ Centre for Water Research, The University of Western Australia, Nedlands, Western Australia 6009
}

BOUALEM BOASHASH

Department of Electrical Engineering, University of Queensland, St. Lucia, Queensland 4067, Australia

(Manuscript received 15 July 1985, in final form 22 April 1986)

\section{ABSTRACT}

\begin{abstract}
The Wigner-Ville distribution, a new tool in the time-frequency analysis of signals, is applied to temperature gradient microstructure records. In particular, the Wigner-Ville distribution is used to compute the local instantaneous and maximum frequencies of the signal as a function of depth, and these frequencies are then related to the dissipation of turbulent kinetic energy. The method is applied to two temperature gradient microstructure records from the Wellington Reservoir. It is shown that a high resolution estimate of the dissipation is obtained that provides insight into the patchiness, the wavenumber content, and the Reynolds-Froude number variability of the integral scales of motion in a strongly stratified water column.
\end{abstract}

\section{Introduction}

Improved instrumentation has led, in the last ten years, to an increased effort in the study of turbulent microstructure in the ocean, lakes and reservoirs. $\mathrm{Nu}$ merous techniques have been developed which allow estimation of some of the terms in the turbulent kinetic energy budget. Osborn (1980) was the first to estimate the energy dissipation directly from measurements of the velocity fluctuation spectrum (see also Oakey, 1982). Thorpe (1977) introduced the concept of a displacement scale and used it to estimate the dissipation indirectly. This indirect technique has been further developed by Dillon (1984) and Imberger (1985).

An alternative method was developed by Caldwell et al. (1980, 1981), Dillon and Caldwell (1980) and Caldwell and Dillon (1981), who focused on the tracer gradient spectrum. These authors proposed the use of the temperature gradient microstructure spectrum and its comparison to the theoretical spectrum derived by Gibson and Schwarz (1963). For homogeneous isotropic turbulence, the one-dimensional spectrum function has the form

$$
S(k)=\left(\frac{q}{2}\right)^{1 / 2} \frac{\chi f(\alpha)}{k_{B} D}
$$

where $k$ is the wavenumber $\left(\mathrm{rad} \mathrm{m}^{-1}\right), k_{B}$ the Batchelor wavenumber $\left(\epsilon / \nu D^{2}\right)^{1 / 4}\left(\mathrm{rad} \mathrm{m}^{-1}\right), D$ the thermal diffusivity $\left(\mathrm{m}^{2} \mathrm{~s}^{-1}\right), \nu$ the kinematic viscosity $\left(\mathrm{m}^{2} \mathrm{~s}^{-1}\right), \epsilon$

* On leave at the Keck Laboratory, California Institute of Technology, Pasadena, CA 91125. the energy dissipation $\left(\mathrm{m}^{2} \mathrm{~s}^{-3}\right), q$ the rate of strain proportionality constant taken to be 3.4 , and $\alpha$ the nondimensional wavenumber $(2 q)^{1 / 2} k / k_{B}$. The rate of dissipation of temperature variance $\chi$ is defined as $6 D\left\langle\left(\partial T^{\prime} / \partial z\right)\right\rangle, T^{\prime}$ being the temperature fluctuation and the angle brackets denoting an appropriate averaging process. The function $f(\alpha)$ is defined by

$$
f(\alpha)=\alpha\left(e^{-1 / 2 \alpha^{2}}-\alpha \int_{\alpha}^{\infty} e^{-1 / 2 \beta^{2}} d \beta\right) .
$$

The method basically fits the experimental spectral peak and rate of roll-off to that predicted by (2) using the dissipation $\epsilon$ as the free parameter.

The success of all the above techniques hinges on the ability to find subsections of the microstructure record with well-defined stationary properties. The proposed use of the Wigner-Ville distribution is essentially a systematic way of achieving this search. The technique is equally suitable for temperature or velocity gradient signals. In this presentation we shall concentrate on temperature microstructure records.

The temperature gradient microstructure is usually measured using thermistor sensors (Caldwell and Dillon, 1981) that have a time constant of around $11 \mathrm{~ms}$. The signals from such a sensor may be digitally enhanced (Fozdar et al., 1985) to yield a frequency response of around $40 \mathrm{~Hz}$. The optimum travel speed of the sensor is determined by a compromise between "freezing" the turbulence and matching this upper-frequency response to the Kolmogorov scale. Typically, the vehicle speed used ranges around $0.1 \mathrm{~m} \mathrm{~s}^{-1}$. 
In order to compute the spectral density, a fast Fourier transform is required of a subsection of the signal. As is well known (Otnes and Enochson, 1978), increasing the length of the record $T$ increases the resolution in the frequency domain. Band averaging is used to further smooth the spectral estimate in order to improve the statistical stability of the estimate. Band averaging in this way is exactly analogous to limiting the lag of the covariance function in the method of Blackman and Tukey (1958).

In a stationary process, arbitrary statistical stability, at a given frequency bandwidth, may thus be achieved by increasing the record length. However, temperature gradient microstructure is not stationary; the signal may vary over a few tens of centimeters from an active turbulence signature to one characteristic of internal wave motions (Caldwell et al., 1980). Further, as shown by Perry and Chong (1982), even for turbulence in a homogeneous fluid, a single realization must be taken over a record long enough to ensure that all wavelengths of the motion contributing to the physical processes are included. Otherwise, as detailed by Imberger (1985) and Dillon and Park (1986), the choice of length of record and starting time greatly influences the spectral shape and thus the estimate of the mechanical dissipation. Most importantly, it must be remembered (Armi and Flament, 1985) that the information describing the distribution of the signal in time is contained in the phase spectrum, which is discarded in normal spectral time-series analysis. Information concerning the distribution in time of energy is thus completely lost in the spectral energy analysis.

Two competing factors must therefore be noted. First, the turbulence as envisaged in the Batchelor (1959) theory is characterized by a single turbulent kinetic energy dissipation, which is given by a volume average over many Kolmogorov scales. A record must thus be long enough to contain many realizations of the highest strain rates so that it represents an ensemble of strain rate regions. Second, this average dissipation will have its own length scale of variation and the length of record should be short compared to this length scale.

Unfortunately, in a stratified fluid we do not, a priori, know the length scale over which the dissipation varies, and so a spectral analysis with a fixed record length algorithm will often include turbulence signatures from a number of different populations (different mean dissipation) or be out of phase with the boundaries separating different populations.

For a particular length of record for a particular realization, the problem of nonstationarity may be clarified by example. Spectral analysis assumes that any signal may be represented by the idealization

$$
x(t)=\sum_{n=1}^{N} a_{n} e^{i \omega_{n} t^{t}}
$$

The Fourier transform of this process is given by (Lighthill, 1962)

$$
X(f)=\sum_{n=1}^{N} a_{n} \delta\left(f-\omega_{n}\right),
$$

yielding energy at definite frequencies. However, if the spectral bandwidth, defined by the $3 \mathrm{db}$ point, of the signal is changing with time (or space, as is the case here), reflecting the variability in the energy dissipation, then a better model of a particular realization is given by

$$
x(t)=\sum_{n=1}^{N} a_{n} e^{i \omega_{n} t} \Pi_{T_{n}}\left(t-n T_{n}\right),
$$

where $T_{n}$ is the length of the time interval over which the spectral bandwidth is constant, and $\Pi_{T_{n}}$ is the boxcar function of length $T_{n}$. The Fourier transform of (5) is given by (Lighthill, 1962)

$$
X(f)=\sum_{n=1}^{N} \frac{\sin \pi\left(f-\omega_{n}\right) T_{n}}{2 \pi\left(f-\omega_{n}\right)} e^{-2 \pi i\left(f-\omega_{n}\right) n T_{n}}
$$

In this "patchy" model, spectral energy is "leaked" into neighboring frequencies, and the location of a particular record interval (with frequency $\omega_{n}$ ) is completely specified by the phase of (6). Comparison of (6) with a known theoretical spectrum derived under the assumption of a single energy dissipation (single frequency in this model) thus leads to severe errors in the dissipation estimate as well as a lack of definition of the variability within the record.

Techniques have been developed to estimate the instantaneous frequency of a gliding tone signal defined as the frequency of that Fourier component of the tone for which the phase is stationary with respect to variations of frequency at the given instant. The time at which the instantaneous frequency of a gliding tone (chirp signal) takes a certain value may be determined by passing the tone through a narrowband filter and noting the instant of maximum response. Barber and Ursell (1948) showed that the response of the filter is sharpest and the peak signal-to-noise ratio of its output the greatest if its bandwidth is set equal to the square root of the rate of change of the frequency.

These results may be generalized and applied to the computation of the spectral density since the shorttime Fourier transform is equivalent to a band-limited filter. This may be seen by examining the short-time Fourier transform of a gliding tone (chirp signal).

The short-time energy spectrum (STES) of a signal $z(t)$ is defined as

$$
E(t, f)=\left|\int_{t-\Delta / 2}^{t+\Delta / 2} z(\theta) e^{-i 2 \pi f \theta} d \theta\right|^{2}, \quad,
$$

where $\Delta$ is the length of record. It represents the spectral energy of a slice of signal of width $\Delta$, calculated around time $t$. by

The linear FM signal $z(t)$ of duration $T$ is expressed

$$
z(t)=\Pi_{T}(t) e^{i \Phi(t)},
$$


where the phase

$$
\Phi(t)=2 \pi\left(f_{0} t+\frac{B}{T} \frac{t^{2}}{2}\right),
$$

and $B$ represents the bandwidth of the signal. The instantaneous frequency is defined by (Ville, 1948)

$$
f_{i}=\frac{1}{2 \pi} \frac{d \Phi}{d t}
$$

The STES of the chirp signal $z(t)$ is given by Papoulis (1973) and Bouachache (1978b):

$$
\begin{aligned}
E(t, f)=\frac{T}{2 B}\left\{\left[C\left(x+\frac{\delta}{2}\right)-C\left(x-\frac{\delta}{2}\right)\right]^{2}\right. & \\
& \left.+\left[S\left(x+\frac{\delta}{2}\right)-S\left(x-\frac{\delta}{2}\right)\right]^{2}\right\},
\end{aligned}
$$

for $-T / 2 \leqslant t \leqslant T / 2$,

where

$$
x=\left(\frac{2 T}{B}\right)^{1 / 2}\left[f_{i}(t)-f\right] ; \quad \delta=\left(\frac{2 B}{T}\right)^{1 / 2} \Delta,
$$

and $C$ and $S$ represent the cosine and sine Fresnel integrals. The influence of the parameter $\delta$ on $E(t, f)$ is shown graphically in Fig. 1, where it is seen that the STES represents a main lobe around the instantaneous frequency $f_{i}(t)$ and that the resolution of the method is determined by the width of this lobe. Figure 1 indicates that the resolution is fixed by the parameter $\delta$ and therefore by the choice of the width $\Delta$. If the width is too large, the main lobe of the energy spectrum is widened to the "large spectral content" within the slice. On the other hand, if the width is too small, the main lobe is also widened, as a consequence of the uncertainty relations (Gabor, 1946).

The optimum width corresponds to the best compromise. We find graphically that the optimum is given by $\delta=(2)^{1 / 2}$ corresponding to

$$
\Delta=\left(\frac{T}{B}\right)^{1 / 2}=\left|\frac{d f_{i}}{d t}\right|^{-1 / 2} .
$$

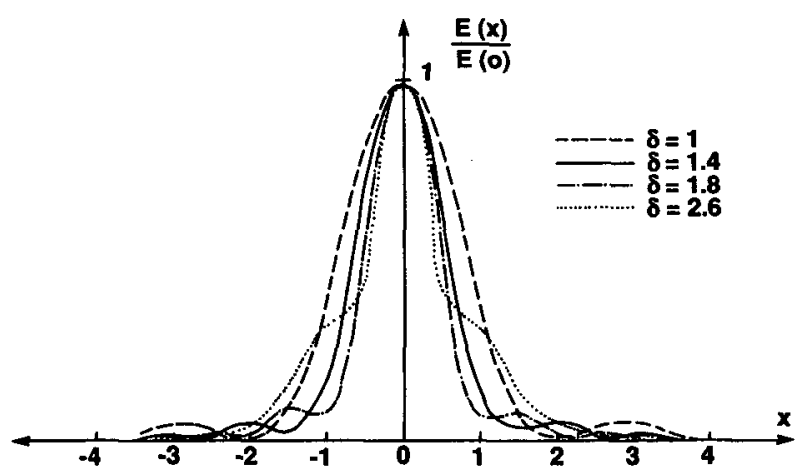

FIG. 1. The normalized energy spectrum of the chirp signal for different values of the parameter $\delta$.
This result implies that the constancy of the instantaneous frequency may be used as a discriminator in the search for the optimum subrecord lengths for nonstationary signals. In other words, an optimum spectral analysis can only be obtained with the spectrogram by choosing a variable window length. This requires an a priori knowledge of $f_{i}(t)$, information that can only be obtained from a time-frequency analysis. An alternative way of carrying out the analysis is to execute a sliding short-time Fourier transform and pass the record through the algorithm, each time changing the window length. The optimum window is achieved when the variability of the dissipation estimate matches the window length. However, such an algorithm is computationally unattractive.

To overcome the above constraints of the spectral methods, the concept of time-frequency distributions was introduced by Gabor (1946), Page (1952), Blanc Lapierre and Picinbono (1955), Priestley (1965), Bouachache (1978a, 1979b), Escudie (1979), Claasen and Mecklenbrauker (1980) and Friedman (1985). These methods take into account variation in both time and frequency and allow the determination of the distribution and evolution of the energy of the observed phenomenon in a time-frequency domain.

Several time-frequency distributions have been proposed, and a comparative study by Bouachache (1979b) has shown that the Wigner-Ville distribution is theoretically the best tool for a time-frequency signal analysis. It does not depend on any window width defined a priori (and can be interpreted as an autoadaptative frequency demodulation) and exhibits the best resolution in the time-frequency domain around the true time-frequency law of the signal (see also Bouachache, 1979a). Bouachache (1978a) successfully implemented the technique and applied it to emitted chirp signals used in the study of the dispersion and absorption of acoustic signals within the earth.

The present work is an adaptation of the above algorithm to particular microstructure realizations and an attempt to give the Wigner-Ville distribution a simple physical interpretation. The technique is shown to yield useful insight into the spatial variation of the temperature gradient field at scales previously hidden by the record length considerations of the spectral density techniques. Attention is focused completely on particular realizations of the microstructure, and emphasis is placed on the understanding of particular records, rather than on the statistics of many records.

\section{Definition of the Wigner-Ville distribution and its relationship to the derivative of the energy spec- trum}

The Wigner-Ville distribution is defined as (Wigner, 1932; Ville, 1948)

$$
W_{z}(t, f)=\int_{-\infty}^{\infty} z\left(t+\frac{\tau}{2}\right) z^{*}\left(t-\frac{\tau}{2}\right) e^{-i 2 \pi f \tau} d \tau,
$$


where $z(t)$ is the analytic signal associated with the real signal $x(t)$. It is seen that $W_{z}(t, f)$ is the Fourier transform of the kernel $z(t+\tau / 2) z^{*}(t-\tau / 2)$ with respect to the variable $\tau$. The instantaneous frequency is then given by

$$
f_{i}(t)=\frac{\int_{-\infty}^{\infty} f W_{z}(t, f) d f}{\int_{-\infty}^{\infty} W_{z}(t, f) d f} .
$$

The analytic signal is constructed by taking the Fourier transform of the signal $x(t)$, removing all contributions on the negative frequency axis, and then taking the inverse of twice the contribution remaining on the positive axis. The relationship between $x(t)$ and $z(t)$ is easily constructed as follows. Suppose the Fourier transform of $x(t)$ is $X(f)$, then we must find the Fourier inverse of the function $2 X(f) U(f)$, where $U(f)$ is the Heaviside step function. This inverse is, however, just the convolution of the inverse of $X(f)$, which is $x(t)$, and the inverse of $U(f)$, which is given in Lighthill (1962):

$$
U(t)=\frac{1}{2} \delta(t-\zeta)+\frac{i}{2 \pi} \frac{1}{(t-\zeta)}
$$

so that

$$
z(t)=x(t)+\frac{i}{\pi} P \int_{-\infty}^{\infty} \frac{x(\zeta)}{(t-\zeta)} d \zeta,
$$

where $P$ stands for the principal part. It is shown in Titchmarsh (1937) that the imaginary part defines the Hilbert transform of the signal $x(t)$.

Hence,

$$
z(t)=x(t)+i H[x(t)],
$$

where $H[x(t)]$ is the Hilbert transform of $x(t)$. The analytic signal at time $t$ thus depends on the signal in a neighborhood around the point defined by the integral (17).

An alternative time-frequency distribution was proposed by Page (1952):

$$
\rho(t, f)=\frac{\partial}{\partial t}\left|\int_{-\infty}^{t} z(\tau) e^{-i 2 \pi f r} d \tau\right|^{2},
$$

where $z(t)$ is the analytic signal associated with the real signal $x(t)$. This represents the derivative of the energy spectrum and is usually referred to as the instantaneous power spectrum.

Escudie (1979) and Bouachache (1979a) showed that $\rho(t, f)$ is related to $W(t, f)$ as follows:

$$
\rho(t, f)=W_{z}(t, f)_{t, f}^{*} P(t, f),
$$

where ${ }_{t, f}^{*}$ is the two-variable convolution and $P(t, f)$ is the two-dimensional Fourier transform of $p(\eta, \tau)$ defined by

$$
p(\eta, \tau)=e^{+i \pi \eta|\tau|} .
$$

This characteristic function appears when we calculate the two-dimensional Fourier transform of $\rho(t, f)$. From (20),

$$
R(\eta, \tau)=\tilde{W}_{z}(\eta, \tau) p(\eta, \tau),
$$

where $R$ and $\tilde{W}_{z}$ are the two-dimensional Fourier transform of $\rho$ and $W$ and $\eta$ and $\tau$ are the independent Fourier variables. From (21) and (22) it follows that

$$
\tilde{W}_{z}(\eta, \tau)=R(\eta, \tau) e^{-i \pi \eta|\tau|} .
$$

Taking the two-dimensional Fourier transform of (23) leads to the result

$$
W_{z}(t, f)=\rho(t, f)_{t, f}^{*} g(t, f),
$$

where $g(t, f)$ is the inverse two-dimensional Fourier transform of $e^{-i \pi \eta|\tau|}$, given by $4 \cos (4 \pi f t) U(t)$, and $U(t)$ is the Heaviside step function. The Wigner-Ville distribution is thus the filtered instantaneous power spectrum.

The analytic signal is used throughout for two reasons. First, by introducing the imaginary part of the signal we define the phase, and second, the use of the analytic signal, distinct from the real signal, eliminates low-frequency artifacts in $W_{z}(t, f)$ that are otherwise created by the interference of the energy spectrum from the negative part of the frequency axes with those from the positive side. As seen from property 1 (Appendix) removing the component of the energy spectrum from the negative axis eliminates this interference (Boashash, 1985).

\section{Computation of the Wigner-Ville distribution}

The analytic signal is first calculated in the frequency domain by computing the fast Fourier transform of the signal, discarding the values for negative frequencies, and then inverting the results in accordance with (18). For computational convenience the record was divided into sections of length $T$ equal to $0.512 \mathrm{sec}$ in order to calculate the analytic signal. This yielded acceptable results since the sampling frequency of 100 $\mathrm{Hz}$ (folding frequency $50 \mathrm{~Hz}$ ) yielded product $B T$ $(=250)$ greater than 10 (Bouachache, 1979b). The Wigner-Ville distribution was then computed by taking the fast Fourier transform with respect to $\tau$ of the kernel

$$
S(t ; \tau)=w(\tau) z\left(t+\frac{\tau}{2}\right) z^{*}\left(t-\frac{\tau}{2}\right),
$$

where $w(\tau)$ is a cosine-bell filter applied to minimize energy leakage. In order to preserve the amplitude of the result, the filter weights were chosen such that

$$
\frac{1}{T} \int_{0}^{T} w(\tau) d \tau=1
$$

where $T$ is the window length.

\section{Examples of test signals}

A number of test signals have already been presented by Page (1952) and Claasen and Mecklenbrauker 
(1980). However, it is convenient here to discuss one of their trial signals and present a more unified solution in order to illustrate the connection between the Wigner-Ville distribution and the instantaneous power spectrum. Following this, two further test signals are used to identify the frequency and time resolution of the numerically computed Wigner-Ville distributions.

Consider first the test signal

$$
x(t)=\cos 2 \pi \omega t .
$$

The Fourier transform $X(f)$ is given by

$$
X(f)=\frac{1}{2}[\delta(f+\omega)+\delta(f-\omega)] .
$$

Eliminating the values for negative frequency and inverting leads to the following expression for the analytic signal:

$$
z(t)=e^{i 2 \pi \omega t} .
$$

It is noteworthy that the real part is the same as the original signal. Substituting the analytic signal (29) into (14) leads to the following expression for the WignerVille distribution:

$$
W(t, f)=\delta(f-\omega),
$$

which is no longer a function of time.

The instantaneous power spectrum is most conveniently computed from the expression

$$
g_{t}(f)=\int_{-\infty}^{t} z(\tau) e^{-2 \pi i f r} d \tau .
$$

Substituting (29) into (31) yields

$$
g_{t}(f)=\frac{1}{2} \delta(f-\omega)+\frac{\sin 2 \pi(\omega-f) t}{2 \pi(\omega-f)}-i \frac{\cos 2 \pi(\omega-f) t}{2 \pi(\omega-f)} .
$$

The value of the instantaneous power spectrum then follows directly from (19) and (32):

$$
\rho(t, f)=\delta(f-\omega) \text {. }
$$

This is the same as the Wigner-Ville distribution, but it also follows directly that

$4 \int_{-\infty}^{\infty} \int_{-\infty}^{\infty} \delta(f-\zeta-\omega) \cos (4 \pi \zeta \tau) U(\tau) d \zeta d \tau=\delta(f-\omega)$,

illustrating that in this case the Wigner-Ville distribution, the instantaneous power spectrum and the simple energy spectrum are all identical. In general, this is not the case.

The chirp signal defined by (9) may be rewritten in the form

$$
x(t)=\cos \pi \omega\left(t-t_{0}\right)^{2},
$$

and it is a convenient tool to see the advantages of the Wigner-Ville distribution over that of spectral methods. The instantaneous frequency $f_{i}(t)$ is given by $(10)$ :

$$
f_{i}(t)=\omega\left(t-t_{0}\right)
$$

This signal was processed to compute the Wigner-Ville distribution, and the results are shown in Fig. 2. The linear spectrum clearly shows the presence of energy from about 5 to $45 \mathrm{~Hz}$. The Wigner-Ville distribution, however, is able to separate the frequency contribution as a function of time, and it is seen that the distribution is a smoothed sinc function centered around the instantaneous frequency given by (36). There is some evidence of a start and end effect, but by choosing the cosine bell to extend over the actual window these effects are kept to a minimum. The algorithm is thus successful at revealing the frequency content at any particular time.

An example to assess the temporal resolution of the algorithm is provided by the function

$$
x(t)=\sin 2 \pi h(t)
$$

where

$$
h(t)=f_{0} t+f_{1} b \arctan \left(\frac{t-t_{0}}{b}\right),
$$

and $f_{0}, f_{1}, b$ and $t_{0}$ are constants. The instantaneous frequency of this signal is again given by (10):

$$
f_{i}(t)=f_{0}+\frac{f_{1}}{1+\left[\left(t-t_{0}\right) / b\right]^{2}} .
$$

Hence, the frequency reaches a maximum at $t_{0}$ and decreases for larger and smaller times. The rate of decrease is measured by the constant $b$. The WignerVille distributions for three different window sizes are shown in Fig. 3a-c. The constants were chosen to reflect the likely range of frequencies encountered in a typical temperature gradient microstructure signal; informa-

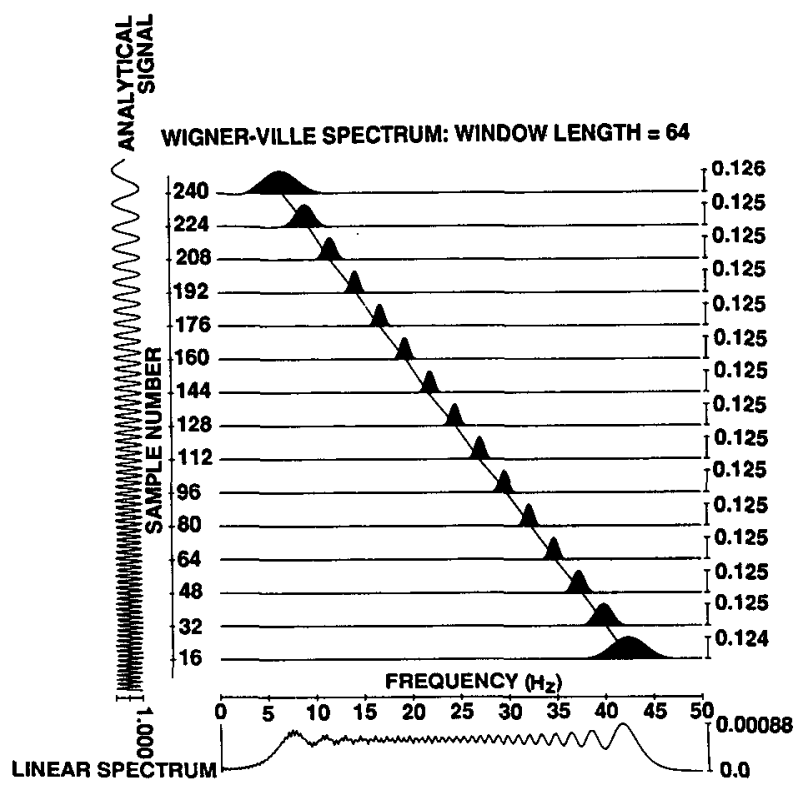

Fig. 2. The Wigner-Ville distribution for the chirp signal $x(t)$ $=\cos \pi \omega\left(t-t_{0}\right)^{2}$, where $\omega=-15.625$ and $t_{0}=+2.88 \mathrm{~s}$. The solid line is the instantaneous frequency. 

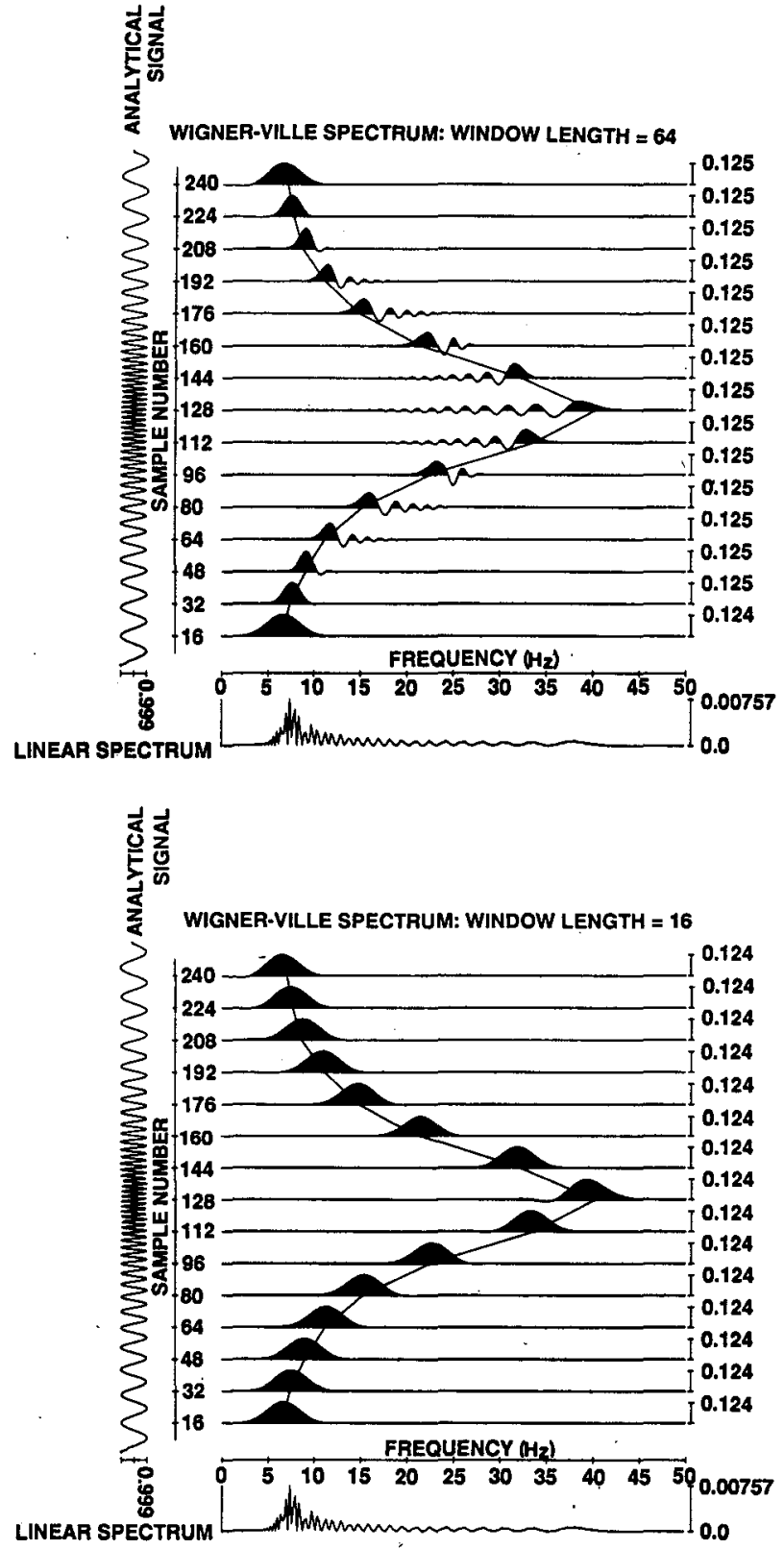

tion below $5 \mathrm{~Hz}$ seldom contributes to the Batchelor spectrum, and information above $45 \mathrm{~Hz}$ is unattainable with present thermistor technology (see Caldwell and Dillon, 1981). It is seen from these results that the rapid change in frequency produces cross terms that are felt about $0.16 \mathrm{sec}$ either side of the frequency maximum for the $0.64 \mathrm{sec}$ window and $0.08 \mathrm{sec}$ for the $0.32 \mathrm{sec}$ window, but without any loss in amplitude in the smaller window. As expected from property $8 \mathrm{ii}$ (Appendix) the instantaneous frequency is not affected by the window length. The results with the smallest window are shown in Fig. 3c. The window corresponds to a spatial resolution of $0.016 \mathrm{~m}$ since the microstructure profiler moves, in our case, with a vertical velocity of

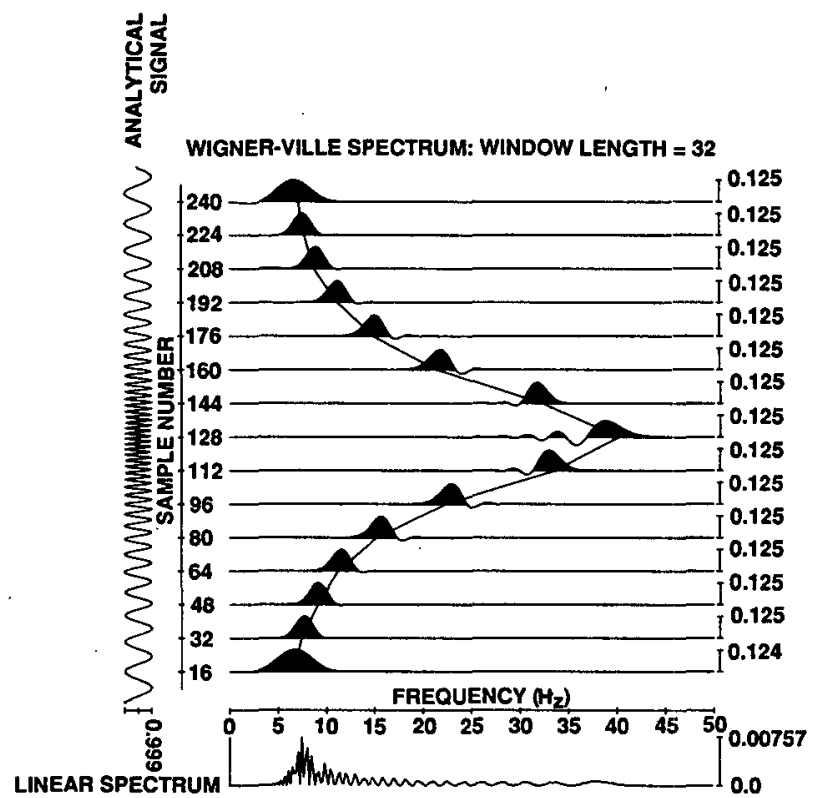

FIG. 3. The Wigner-Ville distribution for the test signal $x(t)$ $=\sin 2 \pi h(t)$, where $h(t)=4.0 t+36.0 \times 0.32 \arctan [(t-1.28) / 0.32]$. The frequency tracking is excellent, but energy leakage is apparent near peak frequency. Results are for a window of (a) $0.64 \mathrm{~s}$, (b) 0.32 $\mathrm{s}$, and (c) $0.16 \mathrm{~s}$. In (b) frequency resolution has not been impaired, but leakage has been reduced. Deterioriation of the frequency resolution in (c) is noticeable at all frequencies.

$0.1 \mathrm{~m} \mathrm{~s}^{-1}$. The smallest window, however, also causes a general smoothing over the whole frequency range, removing the cross terms. The window size for microstructure records with the best compromise between time and frequency resolution thus appears to be 0.32 sec.

Problems may arise in the computation of the instantaneous frequency, as is illustrated by the following example:

$$
x(t)=\cos 2 \pi \omega_{1} t-\cos 2 \pi \omega_{2} t .
$$

It is not difficult to show that the analytic signal is given by

$$
z(t)=e^{2 \pi \omega_{1} t}-e^{2 \pi \omega_{2} t},
$$


resulting in the Wigner-Ville distribution:

$$
\begin{aligned}
W(t, f)=\delta\left(f-\omega_{1}\right)+\delta\left(f-\omega_{2}\right) & \\
& -2 \delta\left(f-\frac{\omega_{1}+\omega_{2}}{2}\right) \cos 2 \pi\left(\omega_{2}-\omega_{1}\right) t .
\end{aligned}
$$

The instantaneous frequency follows from the definitions (15):

so that

$$
f_{i}=\frac{\left(\omega_{1}+\omega_{2}\right)\left[1-\cos 2 \pi\left(\omega_{2}-\omega_{1}\right) t\right]}{2\left[1-\cos 2 \pi\left(\omega_{2}-\omega_{1}\right) t\right]},
$$

$$
f_{i}=\frac{\omega_{1}+\omega_{2}}{2}
$$

Both the first moment and the area of the WignerVille distribution are thus zero for certain values of time (at the nodes), and the numerical evaluation of $f_{i}$, at these times, may lead to unstable estimates. A test was inserted into the numerical algorithm for such ill-conditioned points in time; interpolation, as suggested by the results from the example (35), was used to bridge the gaps.

The third term in (41) is a consequence of the nonlinearity of the Wigner-Ville transform and is an illustration of a cross term. In the present study the Wigner-Ville transform is only used as a vehicle to estimate the maximum frequency at which there is energy and the instantaneous frequency, both of which are not strongly influenced by the presence or absence of cross terms. The general formation of cross terms is discussed more comprehensively by Berry (1977).

\section{Application to microstructure temperature gradient signals}

Microstructure data from two profiles, taken in the Wellington Reservoir, were chosen to illustrate the usefulness of the Wigner-Ville distribution as an indicator of the patchiness of the energy dissipation throughout the water column.

The Wellington Reservoir is a medium-size lake (Imberger, 1985) south of Perth, Western Australia. In February 1985, a ten-day intensive investigation was completed of gravitational adjustments induced by spatially varying surface heating and cooling. The results from the study will appear elsewhere. The particular profiles chosen were taken from this dataset and were collected on the afternoon (1748 and 1757 LST) of Monday, 25 February 1985, near the center of the main reservoir valley approximately $300 \mathrm{~m}$ from the dam wall.

The data were collected from the research vessel, Djinnang $I I$, with the new vertically rising microstructure profiler (RMP) described in Carter and Imberger (1986). Briefly, the profiler is equipped with two FPO7 fast-response thermistors spaced at a distance of 25 $\mathrm{mm}$. Adjacent (within $3 \mathrm{~mm}$ ) to each thermistor is a microconductivity sensor (Head, 1983), allowing measurement of the density microstructure. The ve- hicle rises at $0.1 \mathrm{~m} \mathrm{~s}^{-1}$, with a sampling rate of $100 \mathrm{~Hz}$, through a 16-bit analog-to-digital conversion. There is no umbilical cord; all data is stored internally. Deployment consists of jettisoning the vehicle from the mother ship with a ballast weight. On the downward path the vehicle glides at an angle of approximately $45^{\circ}$ to the vertical, and upon reaching a preset depth, the microprocessor releases the ballast and the profiler commences its rise. Upon retrieval, the data is transmitted to the on-board computers, where the signals are corrected for sensor roll-off (Fozdar et al., 1985) and where the adiabatically corrected density and density gradient channels are added to the data file. In the present case, salinity fluctuations added little to the density perturbations. All subsequent computations are then carried out on the enhanced signals. To assist in the assessment of amplifier noise, one of the temperature gradients is redundantly amplified. By calculating the in-phase power between the two channels, a check is obtained on the noise introduced at the amplifier stage. The results from the two casts are shown in Fig. 4a-d.

The first method of computing the energy dissipation was via the now standard spectral technique (Dillon and Caldwell, 1980). The temperature gradient microstructure records were partitioned into subsections, each containing 512 points. The sections were chosen with a 256-point overlap, and the dissipation was computed by matching the theoretical spectra given by (1) to the computed short-time spectra by adjusting the dissipation that formed the free parameter. The values so obtained from thermistor number 1 are shown in Figs. $5 \mathrm{a}$ and $5 \mathrm{~b}$ for the two profiles.

The second technique involved computing the maximum frequency of the Wigner-Ville transform. The maximum frequency $f_{m}$ was obtained by choosing the maximum frequency at which the Wigner-Ville distribution reached $1 / 10$ of the absolute maximum of the distribution value over the complete frequency bandwidth. Given the interpretation of the Wigner-Ville distribution as the smoothed instantaneous power spectral density (24), this frequency represents the maximum frequency at which the energy budget is adjusting and so is directly related to the smallest scales of the temperature fluctuations:

where

$$
\lambda_{\theta}=\beta \eta_{\theta},
$$

$$
\eta_{\theta}=\left(\frac{\nu D^{2}}{\epsilon}\right)^{1 / 4}
$$

is the Batchelor length scale (Batchelor, 1959) and $\beta$ is a constant.

Given that the profiler rose with nearly constant velocity $v\left(0.1 \mathrm{~m} \mathrm{~s}^{-1}\right)$, the maximum frequency, measured in cycles per second $(\mathrm{Hz})$, will be related to the above minimum length scale as follows:

$$
f_{m}=\frac{1}{2 \pi} \frac{v}{\lambda_{\theta}}
$$




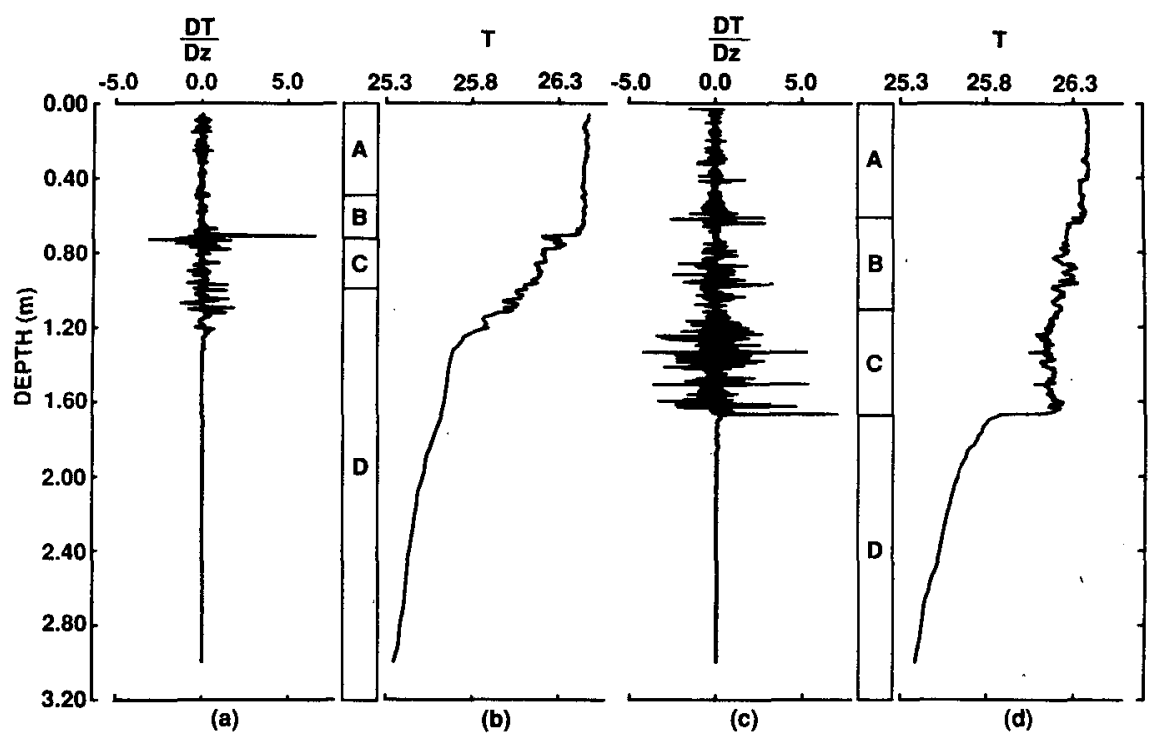

Fig. 4. (a) Temperature microstructure gradient from thermistor number 1 taken at 1748 GMT. Zone A: surface layer; zone B: central mixed layer; zone C: active thermocline; zone D: stratified base. (b) Temperature versus depth from thermistor number 1 taken at 1748 LST. (c) and (d) as in (a) and (b) but for 1757 LST.

Inserting the expression for $\lambda_{\theta}$ from (44) into (46) and solving for the dissipation $\epsilon$ yields

$$
\epsilon=\frac{(2 \pi)^{4} \beta^{4} \nu D^{2} f_{m}{ }^{4}}{v^{4}}
$$

So far, however, we have assumed that the sensor intersects the smallest scales perpendicular to the filament orientation. It may be expected that at these small scales the fluctuations are isotropic and homogeneous (Gibson, 1986a), so that the intersection will, in general, be at an angle of, say, $\theta$ to the vertical. The measured maximum frequency $f_{z m}$ is thus related to the true maximum frequency of the turbulence through the relation

$$
f_{z m}=f_{m} \sin \theta
$$

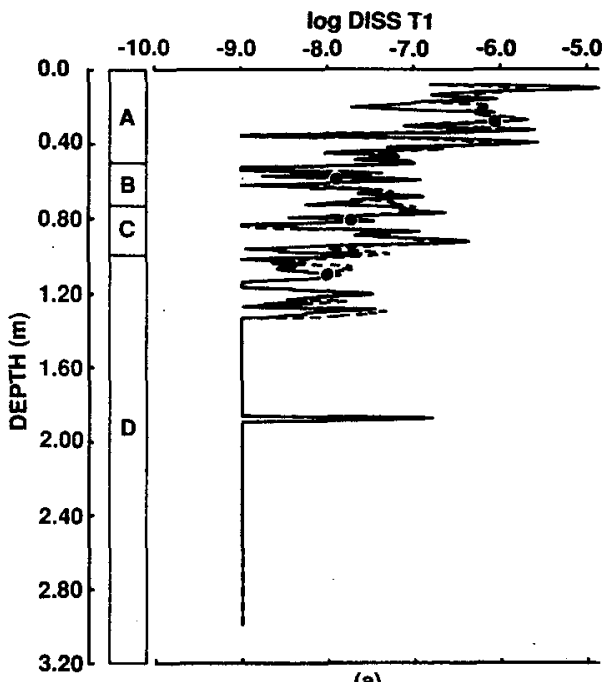

(a)

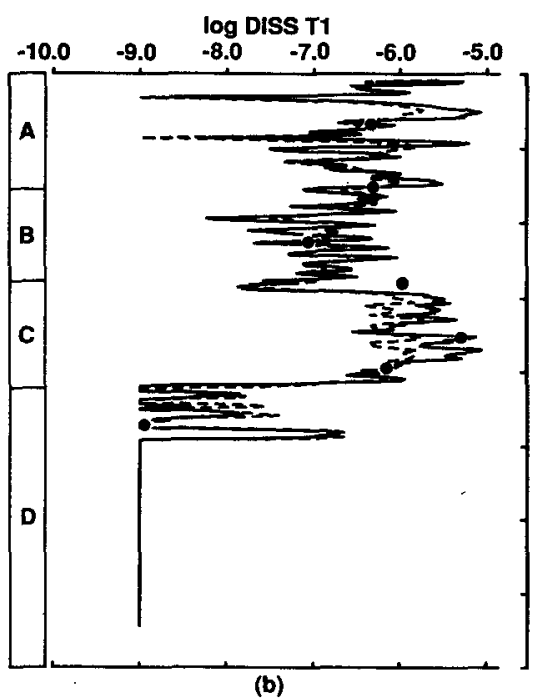

FIG. 5. Logarithm of the dissipation of turbulent kinetic energy versus depth from thermistor number 1 for the profile taken at (a) 1748 and (b) 1757 LST. Dissipation was measured in $\mathrm{m}^{2}$ $\mathrm{s}^{-3}$. Solid circles indicate estimates obtained from the spectral method, solid line indicates estimate obtained from the instantaneous frequency, and dotted line indicates estimate obtained from the maximum frequency. 
If it is now assumed that the probability of any particular orientation being realized is equal, then the average frequency $\overline{f_{z m}}$ for a particular profile is related to the local maximum as follows:

$$
\overline{f_{z m}}=\int_{0}^{2 \pi} \int_{0}^{\pi / 2} f_{m} \sin \theta \frac{d \theta}{\pi / 2} \frac{d \psi}{2 \pi}
$$

where $\psi$ is the azimuthal angle.

Evaluating the integral and substituting the result into (47) yields the final expression for the dissipation in terms of the measured average maximum frequency:

$$
\epsilon=\frac{(2 \pi)^{4} \beta^{4} \nu D^{2}(\pi / 2)^{4}\left(\overline{f_{z m}}\right)^{4}}{v^{4}} .
$$

Equation (50) provides a direct measure of the local dissipation rate once the factor $\beta$ is known. This factor was determined by matching the dissipation values obtained from $(50)$ to those from the spectral method in regions where the dissipation was reasonably constant. The dissipation so obtained (with $\beta=0.699$ ) from thermistor 1 is shown in Fig. 5a and 5b.

The last method investigated involved utilizing the instantaneous frequency as defined by (15). If it is assumed that the instantaneous frequency is a measure of the "frequency content" or the average of the frequency bandwidth at a particular time $t_{0}$, then it is possible to continue the signal on either side of $t_{0}$ with a substitute stationary signal characterized by the same dissipation. With this extension it is then possible, at least intuitively, to assume that the average frequency of the spectral form (2) (given by $\alpha=0.93$ ) is equal to the average of the instantaneous frequency. Since both the measured and theoretical averages are derived for a one-dimensional trace,

$$
\epsilon=\left[\frac{2 \pi(q)^{1 / 2}}{0.93 v}\right]^{4} \nu D^{2} f_{i}^{4}
$$

So far, it has not been possible to prove this intuitively appealing assumption, but the good comparison between the estimate from (50) and (51) (see Fig. 5a and $5 \mathrm{~b}$ ) lends considerable support to this assumption. The latter estimate has the advantage that it is independent of the window length and cross terms of the analysis.

The improved resolution of the two methods based on the Wigner-Ville distribution has brought out the patchy nature of the vertical distribution of the energy dissipation. The changes in the characteristics of the turbulence may now be investigated. This may be done by analyzing the variation with depth of the FroudeReynolds number relationship. In order to demonstrate this relationship, it is convenient to review some simple scaling arguments that characterize the turbulence in the water column. by

Let $N$ be the buoyancy frequency of the fluid defined

$$
N^{2}=-\frac{g}{\rho_{0}} \frac{\partial \rho}{\partial z}
$$

where $\rho$ is the background ambient density of the fluid and $z$ is the vertical coordinate expanded geometrically upward. Further, let $l$ be the vertical scale of an overturn event and $u$ the integral velocity scale. As a patch, defined by a particular overturn event, grows from the time of initiation, the kinetic energy will first be larger than the buoyancy or potential energy inherent in the density profile. However, as demonstrated by Ozmidov (1965), a stage is quickly reached where the two energies balance, so that

$$
N l=u \text {. }
$$

During this initial stage the turbulence "inside" the patch will not be greatly influenced by buoyancy and the Taylor (1935) hypothesis should hold:

$$
u=(\epsilon l)^{1 / 3} \text {. }
$$

Combining (53) and (54) leads to the well-known result:

$$
l=\left(\frac{\epsilon}{N^{3}}\right)^{1 / 2}
$$

as the scale for the maximum height of the overturns. By contrast, the smallest scales of motion $\eta$ and temperature variation $\eta_{\theta}$ are given by the Kolmogorov scales (see Friedlander and Topper, 1962) and Batchelor (1959) scales:

$$
\begin{aligned}
\eta & =\left(\frac{\nu^{3}}{\epsilon}\right)^{1 / 4}, \\
\eta_{\theta} & =\left(\frac{\nu D^{2}}{\epsilon}\right)^{1 / 4} .
\end{aligned}
$$

The associated time scale for the rate of strain is known to be (Gibson and Schwarz, 1963)

$$
\tau=\left(\frac{\nu}{\epsilon}\right)^{1 / 2} .
$$

On the other hand, the time $t_{K}$ taken, after initiation of the overturn event, for the turbulence to become fully developed over the whole range of length scales was deduced by Broadwell and Breidenthal (1982). For large Reynolds numbers this becomes

$$
t_{K}=\frac{l}{u} \text {. }
$$

Substituting the velocity scale from (53) into (59) leads to the simple result:

$$
t_{K}=N^{-1},
$$

which is precisely the time scale of the overturning event itself (Corcos and Sherman, 1976). It is therefore seen that the development of the smallest scales of turbulent motion keeps pace with the growth of the event, and (53) and (54) are consistent.

Once the patch has reached the size given by (55), and in the absence of a continual energy source, the 
larger-scale motions are increasingly influenced by buoyancy forces, and viscous forces progressively erase the smallest scales. This process continues (Gibson, 1982) until the two scales (55) and (56) are equal and the turbulence has essentially decayed. This will occur at a vertical length scale,

$$
\delta=\left(\frac{\nu}{N}\right)^{1 / 2}
$$

Last, it is necessary to introduce the displacement scale $h$, defined as the average vertical excursion of particles at a particular point in the profile (Thorpe, 1977). Different definitions exist, but we define it here as the average of the modulus of the displacement scale calculated from a reordering of the density profile; the reordering was achieved with a bubble-sorting algorithm that monotonizes the density profile.

First efforts at applying the displacement-scale technique to such detailed analysis failed in that it yielded large displacements for the profile taken at 1757 LST at $1.63,1.2,0.7,0.4$ and $0.0 \mathrm{~m}$, these points being the boundaries of the overturning regions (minimum in the instantaneous frequency). The reason for this is easily understood by examining an ideal overturning signature as shown in Fig. 6a. Reordering the profile to make it monotonically increasing leads to the profile shown in Fig. 6b with a corresponding displacement scale shown in Fig. 6c. Since the value used is equal to the distance a particle has moved from the original profile, the largest displacements are calculated for the end boundaries of the event. The normal procedure is to perform heavy filtering on the calculated displacement in order to smooth out these artifically introduced spikes. Alternatively, as suggested by Dillon and Park (1986), the available potential energy can be calculated from the displacement scale and the density anomaly. However, once again, this technique requires averaging over many events. A simple solution is to move the displacement-scale values to the center of the event. This may be achieved by displacing the values up or down by one-half of the displacement scale itself and then taking the average of the result. This results in the distribution shown in Fig. 6d. The displacement scale together with the buoyancy frequency, as calculated for the two profiles for one sensor pair, is shown in Fig. 7.

Given the above scales, it is now possible to investigate the nature of the turbulent motion by defining the Froude and Reynolds numbers of the integral scales of the observed signatures.

The Froude number $\mathrm{Fr}$ is most usefully defined by

$$
\mathrm{Fr}=\frac{u}{N h} \text {. }
$$

Substituting (53) into (62) leads to the interpretation,

$$
\mathrm{Fr}=\frac{l}{h},
$$

which shows that this Froude number may be viewed

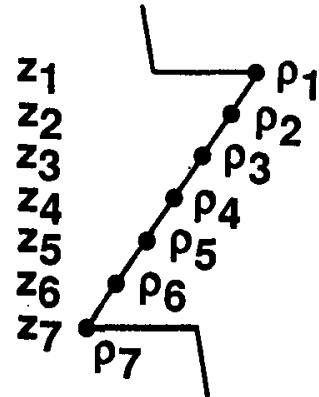

(a)

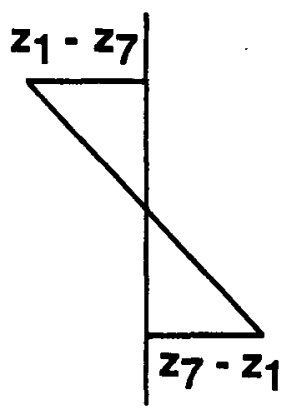

(c)

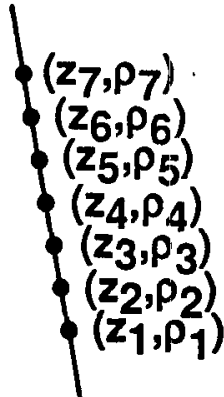

(b)

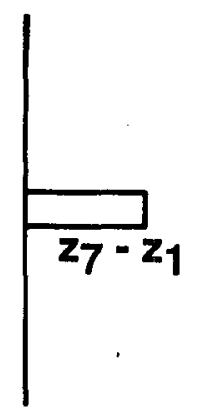

(d)
Fig. 6. Model calculation for the displacement scale. (a) An ideal density profile for an overturn event. (b) The reordered profile. Note changes in original depths $Z_{i}$ to $\bar{Z}_{i}$. (c) The displacement scale $\left(Z_{i}\right.$ $-\tilde{Z}_{i}$ ). (d) The centralized displacement scale.

as the ratio of the Ozmidov length, at the actual dissipation rate, to the measured length scale of the density fluctuations.

This Froude number also has two convenient energy interpretations. First, eliminating the length scale $l$ using (55) leads to

$$
\operatorname{Fr}=\left(\frac{\epsilon}{N^{3} h^{2}}\right)^{1 / 2} \text {. }
$$

This expression shows that the square of this Froude number is the ratio of the energy dissipation to the maximum rate at which the available potential energy $\left(N^{2} h^{2}\right)$ is utilized. Second, from (62) the square of the Froude number is seen to be the ratio of the kinetic energy in the overturn events to the available potential energy in the unstable fluctuations of the density profile. This is why Gibson (1986b) has termed the Froude number the activity parameter.

Lastly, by using the local dissipation result from the Cox model (Osborn and Cox, 1972),

$$
N h^{2} \sim D C,
$$

where $\mathrm{C}$ is the Cox number $\left(\overline{\nabla T^{2}}\right) /(\nabla \bar{T})^{2}$, the Froude number may be written,

$$
\operatorname{Fr} \sim\left(\frac{\epsilon}{D C N^{2}}\right)^{1 / 2}
$$

This last version was first introduced by Gibson (1982). 


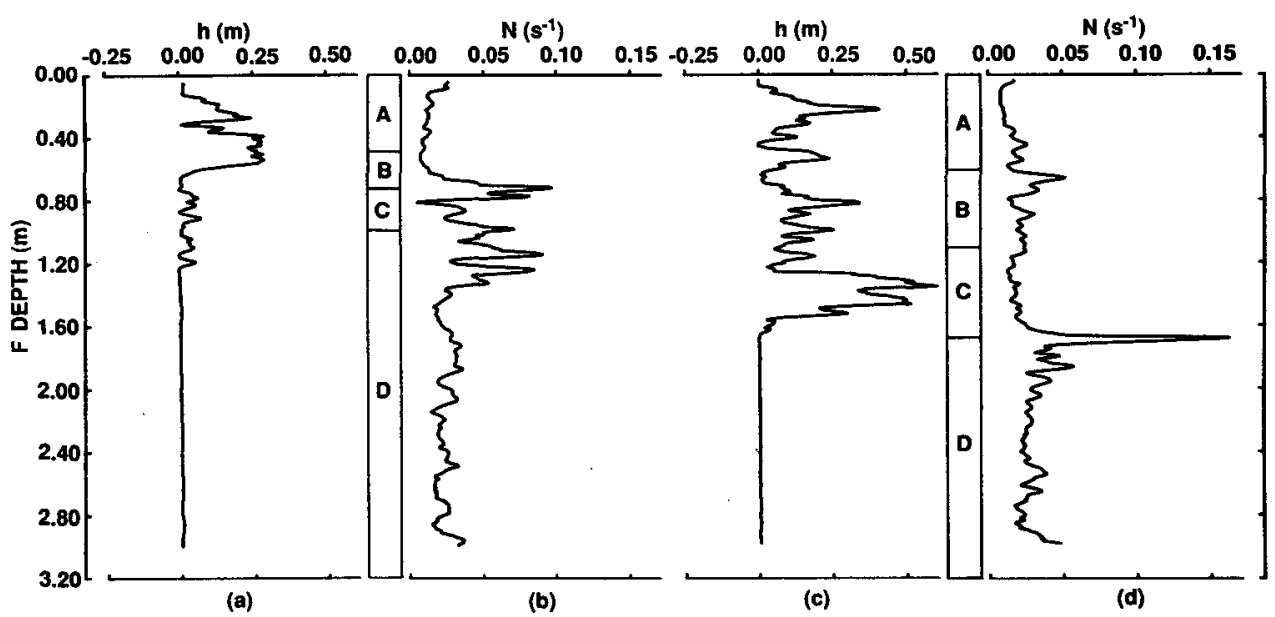

Fig. 7. (a) Centralized displacement scale $h$ calculated for the profile taken at 1748 LST. Density was calculated from the matched temperature and conductivity pair number 1. (b) Buoyancy frequency $N$ calculated from monotonized density for the profile taken at $1748 \mathrm{~h} \mathrm{LST}$. (c) and (d) As in (a) and (b) but for 1757 LST.

Some of the above scaling arguments, in spectral form, have previously been applied by Gibson (1986c) to the experimental work of Stillinger et al. (1983) and Rohr et al. (1984). Gibson showed that in order to have the transition from a buoyancy-dominated flow to an inertial-dominated flow at a unit value of the Froude number, a factor of 1.4 must be introduced such that

$$
\operatorname{Fr}=\frac{1.4 l}{h}
$$

The Reynolds number of the large turbulent motion may also be derived from the above scales:

$$
\operatorname{Re}=\frac{u l}{\nu} \text {. }
$$

Substituting from (53) and (61) leads to

$$
\operatorname{Re}=\left(\frac{l}{\delta}\right)^{2},
$$

and using (55) yields

$$
\operatorname{Re}=\frac{\epsilon}{\nu N^{2}} .
$$

The Reynolds number thus represents the ratio of dissipation to the dissipation at the point where the Ozmidov and Kolmogorov scales are equal. Once again, Gibson (1986a) suggested the introduction of a numerical factor, so that a Reynolds number of unity corresponds to the transition between viscous- and nonviscous-dominated flow. He suggested the following definition:

$$
\operatorname{Re}=\frac{\epsilon}{24.5 \nu N^{2}} .
$$

The only remaining difficulty is the definition of the buoyancy frequency $N$. It should be representative of the buoyancy frequency of the background density gradient before the overturn events introduced instabilities into the profile. Ideally, $N$ should be computed from the monotonic, unstrained original profile over a length scale equal to the overturn length scale $h$. We have chosen to approximate this with $N$ computed from the monotonized profile using a filter with a $3 \mathrm{db}$ point for all variables of $1.6 \mathrm{~Hz}$ (or about $0.06 \mathrm{~m}$ ) in order to obtain maximum resolution.

The data from the two profiles was used to compute both the Froude and the Reynolds number. The results are plotted in Fig. $8 \mathrm{a}$ and $8 \mathrm{~b}$ for the profile taken at 1748 LST and in Fig. 9a and $9 b$ for the data from the profile taken at $1757 \mathrm{LST}$.

\section{Discussion}

The day on which the two profiles were collected had been cloud free and extremely hot with the shortwave radiation reaching about $950 \mathrm{~W} \mathrm{~m}^{-2}$ just after midday. The air temperature reached $42^{\circ} \mathrm{C}$ during midafternoon, and between 0936 and 1545 LST the surface water friction velocity, calculated from the meteorological data corrected for atmospheric stability, was almost constant and below $0.0014 \mathrm{~m} \mathrm{~s}^{-1}$. However, at 1545 LST the wind rose sharply, sending the friction velocity to a peak of $0.0052 \mathrm{~m} \mathrm{~s}^{-1} 30 \mathrm{~min}$ later. Ten minutes after this (1628 LST), the gust had passed and the wind decreased and then remained roughly constant, with a surface friction velocity of $0.0033 \mathrm{~m} \mathrm{~s}^{-1}$, for another $70 \mathrm{~min}$ to $1738 \mathrm{LST}$. At $1738 \mathrm{LST}$ the wind generally decreased to nothing, leaving the water surface once again glassy smooth. Around the time the profiles were taken, from 1748 to $1818 \mathrm{LST}$, the wind decrease temporarily ceased and the wind remained constant with a friction velocity of $0.0018 \mathrm{~m} \mathrm{~s}^{-1}$. The microstructure cast, taken at $1748 \mathrm{LST}$, was thus made at a time of generally decreasing wind, but by the time 

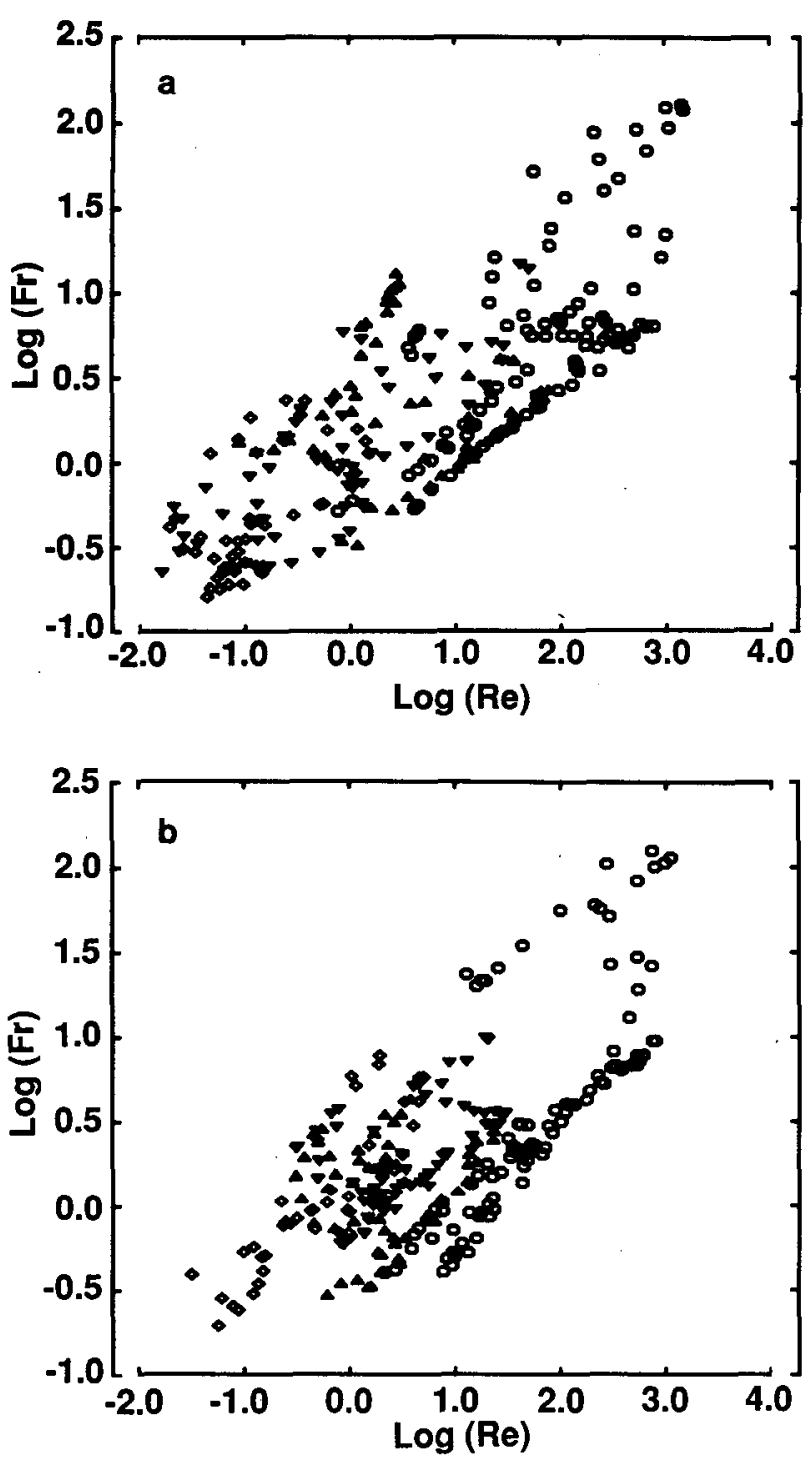

FIG. 8. Froude number versus Reynolds number (a) for sensor pair number 1 and (b) for sensor pair number 2 for the profile taken at $1748 \mathrm{LST}\left(\mathrm{Fr}=1.4 l / \mathrm{h}\right.$ and $\left.\operatorname{Re}=\epsilon / 24.5 v N^{2}\right)$. The symbols have the following meanings: $O$ : data from 0 to $0.48 \mathrm{~m}$ depth (zone $A$ ); $\Delta$ : data from 0.48 to $0.72 \mathrm{~m}$ depth (zone B); $\nabla:$ data from 0.72 to 1.0 $\mathrm{m}$ depth (zone C); and $\diamond$ : data from 1.0 to $3.2 \mathrm{~m}$ depth (zone $\mathrm{D}$ ). The dissipation used here was computed from the instantaneous frequency.

the second cast, at 1757 , was completed, the wind had remained roughly constant for $10 \mathrm{~min}$. At the time of the data acquisition, the incoming solar radiation had fallen to $174 \mathrm{~W} \mathrm{~m}^{-2}$, and the net heat loss due to radiation $\left(130 \mathrm{~W} \mathrm{~m}^{-2}\right)$, to latent heat loss $\left(48 \mathrm{~W} \mathrm{~m}^{-2}\right)$, and to sensible heat gain $\left(10 \mathrm{~W} \mathrm{~m}^{-2}\right)$ was $168 \mathrm{~W} \mathrm{~m}^{-2}$. The water was relatively clear for most of the day with the visible light decreasing to 10 percent of the surface value at a depth of approximately $6 \mathrm{~m}$.

The results from the two casts are shown in Fig. 4. The depth of the mixed layer may be obtained from either the temperature gradient signal (Figs. 4a and 4c) or the temperature curves shown in Figs. $4 \mathrm{~b}$ and $4 \mathrm{~d}$. In the first profile (1748 LST) the depth to which mixing was active was about $1.2 \mathrm{~m}$, and in the second, the depth was $1.6 \mathrm{~m}$, even though the distance between the two profiles was only on the order of $30 \mathrm{~m}$ and the time interval less than $10 \mathrm{~min}$.

It is useful to compute some global parameters before proceeding with a discussion of the signals. For this purpose it is sufficient to assume a mean mixed-layer depth of $1.5 \mathrm{~m}$ and reduced acceleration due to gravity $g^{\prime}$ at the base of the mixed-layer equal to $9.8 \times 10^{-4}$ $\mathrm{m} \mathrm{s}^{-2}$. Using these values, and the given magnitude of the surface fluxes, leads to a surface Monin-Obukhov
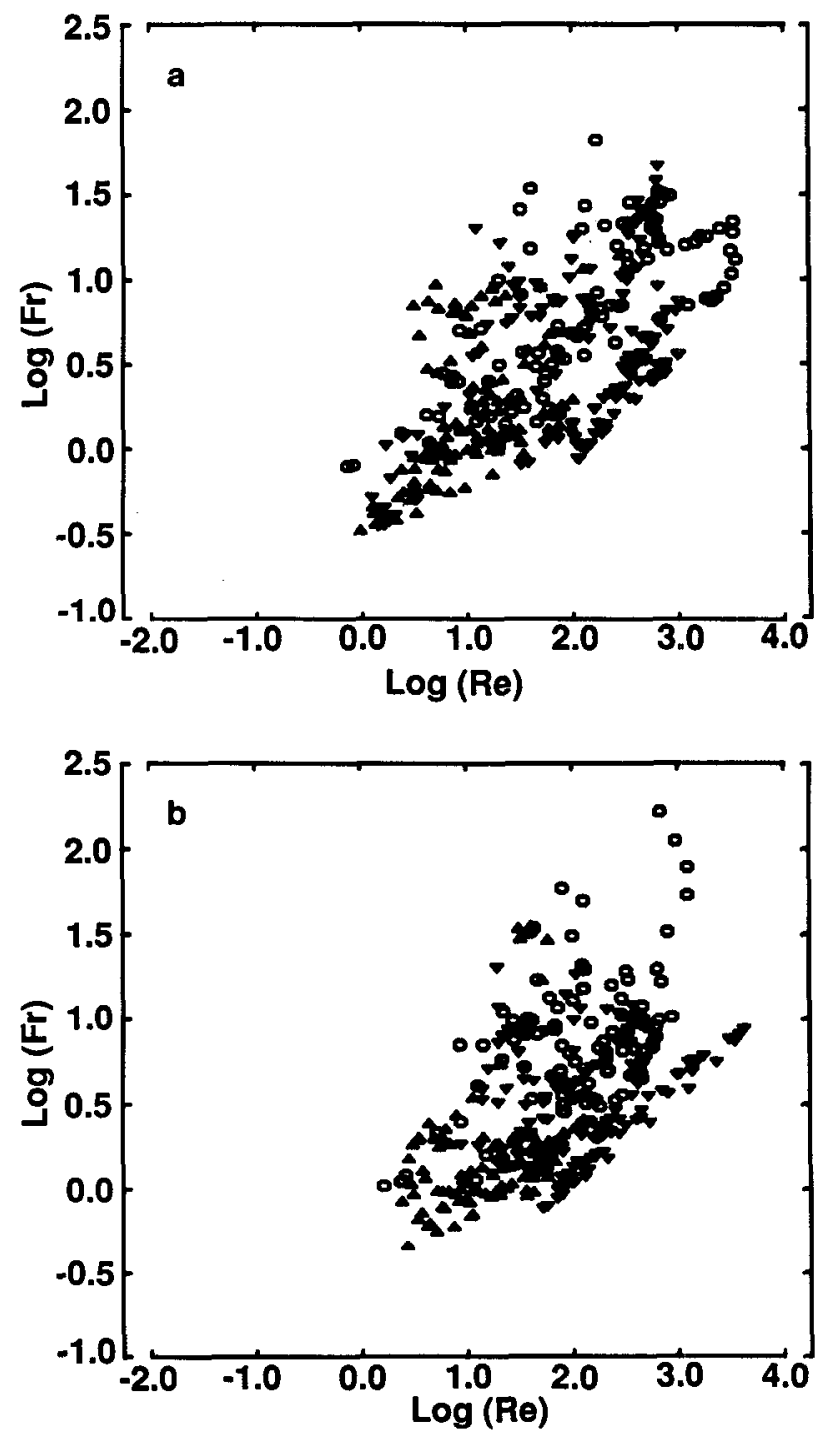

FIG. 9. Froude number versus Reynolds number (a) for sensor pair number 1 and (b) for sensor pair number 2 for the profile taken at $1757 \mathrm{LST}\left(\mathrm{Fr}=1.4 l / \mathrm{h}\right.$ and $\left.\operatorname{Re}=\epsilon / 24.5 \nu N^{2}\right)$. The symbols have the following meaning: $O$ : data from 0.0 to $0.6 \mathrm{~m}$ depth (zone $A$ ); $\Delta$ : data from 0.6 to $1.1 \mathrm{~m}$ depth (zone $B$ ); $\Delta$ : data from 1.1 to $1.7 \mathrm{~m}$ depth (zone C); and $\diamond$ : data from 1.7 to $3.2 \mathrm{~m}$ depth (zone $\mathrm{D}$ ). The dissipation used here was computed from the instantaneous frequency. 
length of $0.06 \mathrm{~m}$ and a Wedderburn number $W=g^{\prime} h^{2}$ $u_{*}^{2} L=0.66$ for a fetch length of approximately 1000 $\mathrm{m}$. Velocity measurements with drogues indicated a general mixed-layer velocity $u$ in the vicinity of 0.02 $\mathrm{m} \mathrm{s}^{-1}$, in good agreement with that computed from a simple momentum balance:

$$
\frac{d}{d t}(u h)=u_{*}^{2}(t)
$$

The billow height to be expected at the base of the mixed layer (Spigel et al., 1986) is $\mathrm{O}\left(u^{2} / g^{\prime}\right)=0.4 \mathrm{~m}$ with a final equilibrium shear zone of about $0.12 \mathrm{~m}$.

The above parameter values therefore allow the data to be put into context as described in Imberger (1985). In the top $0.06 \mathrm{~m}$ or so, the immediate surface production of TKE predominates. Below this, a region of free convection should be discernible to a depth of approximately $0.75 \mathrm{~m}$ (one half of the mixed-layer depth). From 0.75 to $1.2 \mathrm{~m}$ we should find a region energized from above by penetrative convection and from below by production at the base of the mixed layer. However, there is little production itself in this part of the mixed layer. Below this depth and above the base, the turbulence should be strongly influenced by shear production with sporadic billowing periodically covering this region of the water column. Below the base of the mixed layer, the structure should be essentially stable with only internal waves present due to energy leakage through the base.

Inspection of Figs. $4 \mathrm{a}$ and $4 \mathrm{~b}$ essentially confirms these gross predictions. In the profile taken at 1748 LST, an overturn event at the base had introduced an almost linear variation in the density between 0.7 and $1.3 \mathrm{~m}$. Visual inspection of this profile shows fewer high frequency fluctuations, even though the mixing itself was still quite incomplete; there remained a large number of discrete, partially mixed fluid parcels in this profile, similar to that found in the laboratory by Koop and Browand (1979). By contrast, the profile shown in Fig. 4d may be compared to the overturn profiles documented in Imberger (1985). Such comparison leads to the conclusion that the overturn event between 1.2 and $1.63 \mathrm{~m}$ was at the early active stages of development. The profile is unstable and the signal is rich in high wavenumber fluctuations in addition to numerous well-defined large-scale features. In the region between 0.6 and $1.10 \mathrm{~m}$ the profile shows some overturning events originating, most likely, from natural convection penetrating down from the surface. However, the signal once again appeared to contain fewer high-frequency fluctuations in the whole region; the larger unstable parcels were apparently readjusting their vertical positions with minimal mixing under the influence of gravity in a region with only a weak mean vertical density gradient.

In each case, at the upper boundary of these regions a small, but well-defined wisp or billow signature (Imberger, 1985) is observed; they are at $0.7 \mathrm{~m}$ in Fig. $4 \mathrm{~b}$ and at $0.45 \mathrm{~m}$ in Fig. $4 \mathrm{~d}$. The presence of these overturning features means that weak interfaces formed by the overturning processes had been sharpened by the surface processes, and this had given birth to new local shear instabilities. Above these two corresponding signatures, the water column was active, containing increasingly fewer large-scale features as the surface was approached.

These general features were used to divide the profiles into zones, as illustrated in Fig. 4. The data confirm that a surface layer, typical of a stratified shear flow, possesses large-scale features responsible for overturning events. However, these events are intermittent and contain regions of incomplete mixing. Large-scale features persist within overturning events during their whole life, but small-scale fluctuations appear to die out rapidly, leaving unstable, unmixed fluid parcels in the water column.

The above was implied by our knowledge of the mixed-layer dynamics (Imberger, 1985) and of the forcing conditions, such as the value of the Wedderburn number and the ratio of the Monin-Obukhov length to the depth of the mixed layer. However, it remains to be shown that the temperature gradient microstructure is, in general, consistent with the above interpretation.

Consider first the profile taken at 1748 LST (Fig. 4a and $4 \mathrm{~b}$ ). In zone A the surface wind stirring and penetrative convection predominated, leading to a high, but gradually decreasing with depth, value of the dissipation of turbulent kinetic energy. Data from this zone is located in the upper right-hand corner in Figs. $8 \mathrm{a}$ and $8 \mathrm{~b}$, indicating that the turbulence near the surface was essentially unaffected by buoyancy. The consistently high dissipation values near the surface agree with the general picture of a surface source of turbulent kinetic energy and a downward flux of energy.

In zone B (Figs. $4 \mathrm{a}$ and $4 \mathrm{~b}$ ) the dissipation was considerably smaller (by nearly a factor of $10^{2}$ ), reflecting a lack of high wavenumber fluctuations. On the other hand, profile data clearly shows a number of largescale density inversions. Consequently, the data from this region scatters around the origin $( \pm 0.5)$ in the Froude-Reynolds number diagram. This lends support to the idea that the motion was driven by natural convection.

Beneath these surface zones, the profile in Fig. $7 b$ indicates a marked increase in the background density gradient, but there is still evidence of considerable overturning motions. The dissipation levels were somewhat higher, but patchy, in zone C (Fig. 5a), once again confirming the initial diagnosis that this was a region of shear production. The strong variability in this region is reflected in Figs. 8a and 8b, where it is seen that the Froude number ranged from $10^{-1}$ to 10 within the zone, which had a vertical extent of only $0.3 \mathrm{~m}$. Thus, it may be concluded (see also Imberger, 1985) that whenever shear production predominates over turbulent diffusion, the dissipation will be ex- 
tremely patchy, ranging from regions with no mixing to regions where mixing is active.

The data below zone $\mathrm{C}$ was characterized by small values of both the Froude and Reynolds numbers, and at about $1.3 \mathrm{~m}$ from the surface the signal fell below what was considered the noise threshold.

A similar pattern emerges from analysis of the data from the profile taken at 1757 LST. The surface layer, designated by zone $\mathrm{A}$, showed both high and low wavenumber activity. Further, as is seen from Fig. 4d, there was a slight decrease in the temperature as the surface was approached, indicating convective cooling. The dissipation (Fig. 5b) was high, although marked by some distinct low-value spikes located at the smooth steplike changes in the temperature profile.

Zone B was characterized by large events (Fig. 7c), but by a low overall dissipation (Fig. 5b). As expected, most of the data from this region scattered around the origin in Fig. $9 \mathrm{a}$ and $9 \mathrm{~b}$. By contrast, zone $\mathrm{C}$ was an active region with a very large dissipation (Fig. 5b), a small $N$, a relatively large displacement scale, and a wide range on the Froude-Reynolds number plot. Below this, in zone $\mathrm{D}$, most of the data was below the noise threshold.

\section{Conclusion}

The Wigner-Ville distribution was introduced and some of the more important properties of the distribution were described. Examples were given to illustrate the time and frequency resolution of the technique. Further, it was shown that the Wigner-Ville distribution is a representation of the instantaneous power spectral density and thus gives valuable information of the bandwidth of nonstationary signals.

Application to two very different temperature gradient signals, collected in the same water body, demonstrated the usefulness of the Wigner-Ville distribution as a high-resolution method for estimating the local dissipation of turbulent kinetic energy.

Using this technique it was shown that the frequency bandwidth of a temperature gradient signal may vary considerably over only small vertical distances, leading to a nonstationary signal. The mixing from large-scale, shear-driven events was found to be patchy with regions of active and weak mixing separated only by centimeters. The decay of turbulence from the high wavenumber end of the spectrum was also illustrated; the cutoff wavenumber was sometimes very low in the presence of discrete unstable patches. It was found that the Froude-Reynolds number diagram served a useful purpose in the analysis of turbulent signatures, and the results generally agreed with interpretations derived from a knowledge of surface fluxes.

In summary, the instantaneous and maximum frequencies as derived from the Wigner-Ville distribution appear to be excellent objective indicators of the wavenumber content of the signal at a specific time (point in space) and so offer a direct measure of the wavelength of the smaller scales present. For events with high wavenumbers, these frequencies yield a local measure of the dissipation. Use of the Wigner-Ville distribution thus circumvents the difficulties associated with other methods as there is no requirement, a priori, that the turbulent signatures be stationary.

Acknowledgments. The authors would like to thank Geoff Prince for implementing the code and David Pullin for carrying out much of the data processing. Geoffery Carter, George Scolaro and David Elford designed and constructed the new microstructure vehicle. Robert Street, Richard Chapman and Stephen Monismith provided useful criticism of an early draft. Tom Dillon provided numerous suggestions, which led to a much improved manuscript. This work was supported by the Australian Special Research Centre Grants Scheme and the Centre for Environmental Dynamics. This article has Environmental Dynamics Ref. No. ED85-112 and Electrical Engineering Ref. No. P393. The first author would also like to register the hospitality of Caltech as well as the financial support of the Sherman Fairchild program.

\section{APPENDIX}

\section{Properties of the Wigner-Ville Distribution}

The following properties of $W_{z}(t, f)$ are useful and have been proven in Bouachache (1978b; 1982), Claasen and Mecklenbrauker (1980) and Boashash and Escudie (1985). Care must be taken, however, to interpret the results as generalized function sense whenever the original functions are not zero at infinity (Jones, 1966; Schwartz, 1966).

\section{Property 1}

The Wigner-Ville distribution can be expressed symmetrically as follows:

$$
W_{z}(f, t)=\int_{-\infty}^{+\infty} Z\left(f+\frac{m}{2}\right) Z^{*}\left(f-\frac{m}{2}\right) e^{i 2 \pi m t} d m,
$$

where $Z(f)$ represents the Fourier transform of $z(t)$ and $Z^{*}(f)$ is its complex conjugate.

\section{Property 2}

$W_{z}(t, f)$ is real for all values of $t$ and $f$.

\section{Property 3}

Integration of the Wigner-Ville distribution over all frequencies yields the instantaneous power of the signal at time $t$ :

$$
\int_{-\infty}^{+\infty} W_{z}(t, f) d f=|z(t)|^{2}
$$

\section{Property 4}

Integration of the Wigner-Ville distribution over all time yields the spectral density of the signal at frequency $f$ :

$$
\int_{-\infty}^{+\infty} W_{z}(t, f) d t=|Z(f)|^{2}
$$




\section{Property 5}

Integration of the Wigner-Ville distribution over all time and all frequencies yields the energy of the signal

$$
\int_{-\infty}^{+\infty} \int_{-\infty}^{+\infty} W_{z}(t, f) d t d f=\int_{-\infty}^{\infty}|z(t)|^{2} d t=E_{z}
$$

\section{Property 6}

The time-frequency domain of $W(t, f)$ is given by the frequency extent and duration of the signal.

- If $z(t)=0$ for $t<T_{1}$ and $t>T_{2}$, then $W_{z}(t, f)=0$ for $t<T_{1}$ and $t>T_{2}$.

- If $Z(f)=0$ for $f<f_{1}$ and $f>f_{2}$, then $W_{z}(t, f)=0$ for $f<f_{1}$ and $f>f_{2}$.

\section{Property 7}

The Wigner-Ville distribution is time and frequency invariant, which means that shifts in time or frequency of the signal correspond to similar shifts on the WignerVille distribution.

- If $y(t)=z\left(t-t_{0}\right)$, then $W_{y}(t, f)=W_{z}\left(t-t_{0}, f\right)$.

- If $y(t)=z(t) e^{j 2 \pi f_{0} t}$, then $W_{y}(t, f)=W_{z}\left(t, f-f_{0}\right)$.

\section{Property 8}

(i) The first-order moment of the Wigner-Ville distribution yields $f_{i}(t)$, the instantaneous frequency of the signal, and $\tau_{g}(f)$, the time delay:

$$
\begin{array}{r}
f_{i}(t)=\frac{\int_{-\infty}^{+\infty} f W_{z}(t, f) d f}{\int_{-\infty}^{+\infty} W_{z}(t, f) d f}, \\
\tau_{g}(f)=\frac{\int_{-\infty}^{+\infty} t W_{z}(t, f) d t}{\int_{-\infty}^{\infty} W_{z}(t, f) d t} .
\end{array}
$$

If the signal is analytic, then Claasen and Mecklenbrauker $(1980)$ have shown that $f_{i}(t) \geqslant 0$. Other moments may be defined and the reader is referred to the article by Claasen and Mecklenbrauker (1980).

(ii) If $h(t)$ is a continuous window of finite duration such that $h(0)=d h /\left.d t\right|_{t=0}=0$ and if $W_{z}^{h}(t, f)$ represents the windowed Wigner-Ville distribution

$$
W_{z}^{h}\left(t_{1} f\right)=\int_{-\infty}^{\infty} h(\tau) z\left(t+\frac{\tau}{2}\right) z^{*}\left(t-\frac{\tau}{2}\right) e^{-2 \pi i f \tau} d \tau,
$$

then

$$
f_{i}(t)=\frac{\int_{-\infty}^{\infty} f W_{z}^{h}(t, f) d f}{\int_{-\infty}^{\infty} W_{z}^{h}(t, f) d f},
$$

which equals the instantaneous frequency $f_{i}(t)$ of the signal $z(t)$, independent of the window $h(\tau)$ (Boashash et al., 1986). This property is important because it shows that the windowing procedure does not influence the estimate of the instantaneous frequency.

\section{Property 9}

Linear filtering of the signal $z(t)$ directly reflects itself in a linear filtering on the Wigner-Ville distribution of the signal. If $z(t)$ is the input signal to a system with an impulse response $h(t)$ such that

then

$$
y(t)=z(t){ }_{(t)}^{*} h(t)
$$

$$
W_{y}(t, f)=W_{z}(t, f)_{(t)}^{*} W_{h}(t, f),
$$

where ${ }_{(t)}^{*}$ denotes the convolution integral in the time domain.

\section{Property 10}

Similar relationships exist for modulation. If $m(t)$ is a modulation function such that

$$
\begin{gathered}
y(t)=m(t) z(t), \\
Y(f)=M(f) * Z(f),
\end{gathered}
$$

then

$$
W_{z}(t, f)=W_{z}(t, f)_{(f)}^{*} W_{m}(t, f) .
$$

Property 11

Given the Wigner-Ville distribution $W_{z}(t, f)$, we can show that (Claasen and Mecklenbrauker, 1980)

$$
\int_{-\infty}^{\infty} W_{z}\left(\frac{t}{2}, f\right) e^{i 2 \pi f t} d f=z(t) Z^{*}(0) .
$$

This shows that the Wigner-Ville distribution conveys and recovers all of the information contained in the signal.

\section{REFERENCES}

Armi, L., and P. Flament, 1985: Cautionary remarks on the spectral interpretation of turbulent flows. J. Geophys. Res., 50, 11779 11782.

Barber, N. F., and F. Ursell, 1948: The response of a resonant system to a gliding tone. Phil. Mag., 39, 345-361.

Batchelor, G. K., 1959: Small-scale variations of convected quantities like temperature in turbulent fluids. J. Fluid Mech., 5, 113-133.

Berry, M. V., 1977: Semi-classical mechanics in phase space: A study of Wigner's function. Phil. Trans. Roy. Soc., A287, 237-271. 
Blackman, R. B., and J. W. Tukey, 1958: The Measurement of Power Spectra. Dover, 190 pp.

Blanc Lapierre, A., and B. Picinbono, 1955: Sur la notion de spectre instantane de puissance. Publ. Sci. Univ., Alger, Ser. B, 1, 232.

Bouachache, B., 1978a: Representation temps-frequence. Publ. ELFAquitaine, No. 373/78, Pau, 56 pp.

- , 1978b: Representation conjointe temps. frequence. Travail de Fin d'Etudes, Institut de Chimie et de Physique Industrielles, Lyon, $120 \mathrm{pp}$.

,$- 1979 \mathrm{a}$ : Representation temps-frequence. Master's thesis, University of Grenoble, $80 \mathrm{pp}$.

$-1979 \mathrm{~b}$ : Sur la possibilite d'utiliser la representation conjointe en temps et frequence de Ville aux signaux vibrosismeques. Colloque National sur le Traitement du Signal, Gretsi, Nice, 121, 1-6.

- 1982: Representation temps-frequence. Ph.D. dissertation, University of Grenoble, $164 \mathrm{pp}$.

Boashash, B. (previously Bouachache), 1985: On the anti-aliasing and computational properties of the Wigner-Ville Distribution. Proc. IASTED Int. Symp. on Signal Processing, Paris, Int. Assoc. of Science and Technology for Development, 290-293.

- (previously Bouachache), and B. Escudie, 1985: Wigner-Ville analysis of asymptotic signals. Signal Processing, 8, 315-327.

$\longrightarrow$ L. White and J. Imberger, 1986: Wigner-Ville analysis of nonstationary random signals, with application to turbulent microstructure signals. Proc. IEEE Int. Conf. on Acoustics, Speech and Signal Processing, Tokyo, IEEE, 2323-2326.

Broadwell, J. E., and R. E. Breidenthal, 1982: A simple model of mixing and chemical reaction in a turbulent shear layer. J. Fluid Mech., 125, 397-410.

Caldwell, D. R., and T. M. Dillon, 1981: An oceanic microstructure measuring system. Oregon State University Tech. Rep. 81-10, $165 \mathrm{pp}$.

,,-- J. M. Brubaker, P. A. Newberger and C. A. Paulson, 1980: The scaling of vertical temperature gradient spectra. $J$. Geophys. Res., 85, 1917-1924.

Caldwell, D. R., T. M. Chriss, P. A. Newberger and T. M. Dillon, 1981: The thinness of oceanic temperature gradients. J. Geophys. Res., 86, 4290-4292.

Carter, G. D., and J. Imberger, 1986: Vertically rising microstructure profiler. J. Atmos. Oceanic Technol., 3, 462-471.

Claasen, T. A. C. M., and W. F. G. Mecklenbrauker, 1980: The Wigner distribution. Philips Res. J., 35, 217-250.

Corcos, G. M., and F. S. Sherman, 1976: Vorticity concentrations and the dynamics of unstable free shear layers. $J$. Fluid Mech., 73, 241-264.

Dillon, T. M., 1984: The energetics of overturning structures. J. Phys. Oceanogr., 14, 541-549.

- - and D. R. Caldwell, 1980: The Batchelor spectrum and dissipation in the upper ocean. J. Geophys. Res., 85, 1910-1916.

- and M. M. Park, 1986: The available potential energy of overturns as an indicator of mixing in the seasonal thermocline. $J$. Geophys. Res. (in press).

Escudie, B., 1979: Representation temps-frequence. Ann. Telecommun., 35, 101-111.

Fozdar, F. M., G. J. Parker and J. Imberger, 1985: Matching temperature and conductivity sensor response characteristics. $J$. Phys. Oceanogr., 15, 1557-1569.

Friedlander, S. K., and L. Topper Eds., 1962: Turbulence: Classical Papers on Statistical Theory. Interscience, $187 \mathrm{pp}$.

Friedman, D. H., 1985: Instantaneous frequency distribution vs time: An interpretation of the phase structure of speech. Proc. IEEE Int. Conf. on Acoustics, Speech and Signal Processing, Tampa, IEEE, $1-4$.
Gabor, D., 1946: Theory of communication. J. IEE, London, 93, $429-457$.

Gibson, C. H., 1982: Alternative interpretations for microstructure patches in the thermocline. J. Phys. Oceanogr., 12, 374-383.

- 1986a: Oceanic turbulence: Big bangs and continuous creation. J. Physicochem. Hydrodyn. (in press).

_- 1986b: Internal waves, fossil turbulence, and composite ocean microstructure spectra. J. Fluid Mech. (in press).

- 1986c: Fossil turbulence and intermittency in sampling oceanic mixing processes. J. Geophys. Res. (in press).

, and W. H. Schwarz, 1963: The universal equilibrium spectra of turbulent velocity and scalar fields. J. Fluid Mech., 16, 365384.

Head, M. J., 1983: The use of miniature four-electrode conductivity probes for high resolution measurement of turbulent density or temperature variations in salt-stratified water flows. Ph.D. dissertation, University of California, $211 \mathrm{pp}$.

Imberger, J., 1985: The diurnal mixed layer. Limnol. Oceanogr., 30, 737-770.

Jones, D. S., 1966: Generalised Functions. McGraw-Hill, 482 pp.

Koop, C. G., and F. K. Browand, 1979: Instability and turbulence in a stratified fluid with shear. J. Fluid Mech., 93, 135-159.

Lighthill, M. J., 1962: Introduction to Fourier Analysis and Generalized Functions. Cambridge University Press, 72 pp.

Oakey, N. S., 1982: Determination of the rate of dissipation of turbulent energy from simultaneous temperature and velocity shear microstructure measurements. J. Phys. Oceanogr., 12, 256-271.

Osborn, T. R., 1980: Estimates of the local rate of vertical diffusion from dissipation measurements. J. Phys. Oceanogr., 10, 83-89.

-, and C. S. Cox, 1972: Oceanic fine structure. Geophys. Fluid Dyn., 3, 321-345.

Otnes, R. K., and L. Enochson, 1978: Applied Time Series Analysis. Vol. I, Basic Techniques, Wiley and Sons, 449 pp.

Ozmidov, R. V., 1965: On the turbulent exchange in a stably stratified ocean. Atmos. Oceanic Phys., 1, 493-497.

Page, C. H., 1952: Instantaneous power spectra. J. Appl. Phys., 23, 103-106.

Papoulis, A., 1973: Systems and Transforms with Application to Optics. McGraw-Hill, 2nd ed., 474 pp.

Perry, A. E., and M. S. Chong, 1982: On the mechanism of wall turbulence. J. Fluid Mech., 119, 173-217.

Priestly, M. B., 1965: Evolutionary spectra and non-stationary processes. J. Roy. Statist. Soc., B27, 204-237.

Rohr, J. J., E. C. Itsveire and C. W. van Atta, 1984: Mixing efficiency in stably stratified decaying turbulence. J. Geophys. Astrophys. Fluid Dyn., 29, 221-236.

Schwartz, L. 1966: Mathematics for the Physical Sciences. Addison Wesley, $357 \mathrm{pp}$.

Spigel, R. H., J. Imberger and K. N. Rayner, 1986: A model for billowing and deepening of the diurnal mixed layer. Limnol. Oceanogr., 31(33), 533-556.

Stillinger, D. C., K. N. Helland and C. W. van Atta, 1983: Experiments on the transition of homogeneous turbulence to internal waves in a stratified fluid. J. Fluid Mech., 131, 91-122.

Taylor, G. I., 1935: Statistical theory of turbulence. Proc. Roy. Soc. London, A151, 421-478.

Thorpe, S. A., 1977: Turbulence and mixing in a Scottish Loch. Phil. Trans. Roy. Soc. of London, A286, 125-181.

Titchmarsh, E. C., 1937: Introduction to the Theory of Fourier Integrals. Oxford University Press, $394 \mathrm{pp}$.

Ville, J., 1948: Theorie et application de la notion de signal analytique. Cables Trans., A(1), 61-74.

Wigner, E. P., 1932: On the quantum correction for thermodynamic equilibrium physics. Phys. Rev., 40, 748-759. 